\title{
Philosophiques
}

\section{Le monisme épistémologique de la science contemporaine}

\section{Louis Valcke}

Volume 1, numéro 2, octobre 1974

URI : https://id.erudit.org/iderudit/203010ar

DOI : https://doi.org/10.7202/203010ar

Aller au sommaire du numéro

Éditeur(s)

Société de philosophie du Québec

ISSN

0316-2923 (imprimé)

1492-1391 (numérique)

Découvrir la revue

Citer cet article

Valcke, L. (1974). Le monisme épistémologique de la science contemporaine. Philosophiques, 1(2), 3-13. https://doi.org/10.7202/203010ar

\section{Résumé de l'article}

L'auteur part du postulat d'objectivité de la méthode scientifique dans son affirmation d'une distinction radicale entre le sujet qui observe et l'objet observé. Il montre en quoi ce dualisme du sujet et de l'objet a été remis en question dans la physique quantique de Heisenberg et dans la psychologie behavioriste de Watson. Cependant, l'auteur soutient que le dépassement de ce dualisme cartésien par le monisme objectiviste (Monod) ou par le monisme subjectiviste (Eddington) est impossible pour la raison très simple que le monisme est impensable. De fait, le monisme a été pensé, mais dans un langage dualiste. 


\title{
LE MONISME ÉPISTÉMOLOGIQUE DE LA SCIENCE CONTEMPORAINE
}

\author{
par Louis Valcke
}

Le postulat d'objectivité de la méthode scientifique pose certaines conditions épistémologiques, telles que le refus systématique de toute explication par la finalité, la reconnaissance d'un déterminisme essentiel, et l'affirmation d'une distinction radicale entre le sujet qui observe et l'objet observé. Arrêtonsnous à cette dernière condition, celle du dualisme cartésien, qui a été remise en cause par Werner Heisenberg.

Werner Heisenberg est parfaitement conscient de la portée épistémologique et philosophique de sa critique. Il est explicite : «L'ancienne division de l'univers en un déroulement objectif dans l'espace et le temps, d'une part, en une âme qui reflète ce déroulement, d'autre part, division correspondant à celle de Descartes en res cogitans et res extensa n'est plus propre à servir de point de départ si l'on veut comprendre les sciences modernes de la nature ${ }^{1}$. Cet ancien dualisme est rejeté, car maintenant, dit-il, «nous nous trouvons dès l'abord au sein d'un dialogue entre la nature et l'homme, dont la science n'est qu'une partie, si bien que la division conventionnelle du monde en sujet et objet, en monde intérieur et en monde extérieur, en corps et en âme ne peut plus s'appliquer... Pour les sciences de la nature... le sujet de la recberche n'est donc plus la nature en soi, mais la nature livrée à l'interrogation bumaine et dans cette mesure l'homme, de nouveau, ne rencontre ici que lui-même ${ }^{2}$. Il est clair que pour Heisenberg il s'agit de beaucoup plus que

1. W. HEISENBERG, La Nature dans la physique contemporaine. Trad. Karvélis et Leroy, Gallimard, Paris, 1962, p. 34.

2. W. HEISENBERG, op. cit., p. 29. 
d'une confrontation entre deux théories physiques. Il y va de la relation sujet-objet, problème proprement philosophique, et la référence explicite à Descartes est indicative.

Ce renversement de perspective est dû, on le sait, au «principe d'incertitude » découlant de la physique quantique. En plus d'une forme que l'on peut qualifier d'essentielle, car elle résulterait de la nature même des particules subatomiques, ce principe prend également une forme «observationnelle». C'est ce second aspect seulement que je voudrais discuter ici.

Par «forme observationnelle »'entends que l'incertitude ne découlerait pas seulement de la nature de l'objet observé ou observable, mais bien de la relation même entre l'observé, l'observateur et le mode d'observation. C'est ce que Heisenberg appelle la «relation d'indétermination», qu'il décrit comme suit: «On peut mesurer la position (d'une particule atomique), mais alors l'intervention de l'instrument d'observation interdit jusqu'à un certain point de connaitre la vitesse ${ }^{3}$. C'est pour cette raison que la physique contemporaine doit rejeter le «dualisme classique» car «la méthode scientifique, qui choisit, explique et ordonne, admet les limites qui lui sont imposées par le fait que lemploi de la métbode transforme son objet, et que par conséquent, la méthode ne peut plus se séparer de son objet ${ }^{4}$.

Tout en reconnaissant l'importance épistémologique de sa remarque, Heisenberg, sagement, se refuse d'aller plus loin. D'autres, sans doute moins sages, en ont inféré un peu hâtivement que Heisenberg a «démontré » que l'objectivité scientifique est impossible. De là, il n'y a qu'un pas à franchir pour affirmer que la méthode scientifique se détruit d'elle-même, puisqu'elle repose touce entière sur le postulat d'objectivité. On constate, avec un plaisir secret mais évident, le paradoxe d'une science qui, par souci d'objectivité, est conduite à se nier en faveur d'une subjectivité intégrale et inévitable. Tout ceci contribue à la renaissance d'un nouveau courant antirationaliste et subjectiviste qui fait feu de tout bois, surtout, oh ! aimable incohérence, s'il est du bois «scientifique »!

3. W. HEISENBERG, op. cit., p. 47.

4. W. HEISENBERG, op. cit., p. 34. Nous soulignons. 
Voyons de plus près, pourquoi et en quel sens « la méthode - ou l'utilisation de l'instrument - transforme son objet». Le principe d'incertitude, sous cette forme opérationnelle, fut formulé après certaines constatations expérimentales paradoxales. Toutes ces expériences ${ }^{5}$ supposent un appareil permettant d'établir la distribution probable de particules subatomiques. Or, on a toujours constaté que cette distribution s'effectue suivant des lois totalement différentes selon que le dispositif est éclairé ce qui permettrait de «voir ce qui se passe»- ou qu'il n'est pas éclairé - ce qui exclut l'observation directe, et ne permet que l'enregistrement, l'impact d'un électron sur un compteur Geiger par exemple. De telles constatations frappent l'imagination. Elles nous semblent inhabituelles, paradoxales, étranges: elles nous donnent l'impression de nous conduire au bord d'un monde inconnu, où tout est «autre», où plus rien n'est «normal ». Posons la question : y a-t-il mystère ou apparence de mystère? Et si ce n'est qu'apparence de mystère, d'où vient cette apparence?

Imaginons une partie de billard à la tombée du jour. Quelqu'un allume la lumière, au moment précis où une bille est lancée sur sa trajectoire. Que se passera-t-il? Rien, rien d'extraordinaire en tout cas: nous verrons un peu mieux la bille qui, imperturbable et impertubée, continuera, sa trajectoire. Mais supposons que chaque fois que jaillit la lumière, la bille tressaute. Mieux, supposons que le comportement des billes varie si l'éclairage varie, au point que le jeu semblera obéir à des lois différentes selon que nous jouons en plein soleil ou dans la pénombre enfumée d'une salle de café. Notre ahurissement serait complet, nous n'y comprendrions plus rien : le mystère serait là, tangible, devant nos yeux.

Or l'électron se comporte exactement comme nos hypothétiques billes de billard : on comprend que le monde de l'électron nous semble «autre», bizarre, mystérieux, quelque peu terrifiant : on est à deux doigts de la science-fiction. Et pourtant, à

5. La description la plus simple et, vue la personnalité de l'auteur, la plus fidèle que je connaisse est donnée par $\mathrm{R}$. Feynman, La nature des lois pbysiques, Marabout, 1971, p. 158 et ss. 
y regarder de plus près - mais heureusement nous ne pouvons pas y regarder de plus près - nos billes de billard se comportent comme l'électron: elles tressautent quand on allume la lumière, mais elles tressautent imperceptiblement. Lorsque la lumière jaillit, des milliards de photons se déchaînent et frappent la bille, qui dévie nécessairement de sa trajectoire. Seulement, l'énergie de quelques milliards de photons, par rapport à la masse inerte de la bille, ce n'est presque rien, et la déviation de la trajectoire, réelle cependant, est bien inférieure au seuil de perception de l'instrument le plus délicat.

Mais dans un dispositif qui permettrait de «voir» la trajectoire d'une particule subatomique, les choses changent, parce que les proportions changent. Si nous voulons voir un électron il faudra l'illuminer, c'est-à-dire projeter sur lui un faisceau lumineux. Mais un faisceau lumineux c'est un flux de photons, et un photon a une énergie comparable à celle d'un électron. L'électron «observé » est un électron qui aura reçu l'impact d'un photon, et dont, par conséquent, la trajectoire aura été modifiée autant que peut l'être celle d'une bille de billard sous l'impact d'une autre bille de billard. Un électron dont la trajectoire n'aura pas été modifiée est un électron qui n'aura pas été touché par un photon, mais qui, par conséquent, sera inobservable. L'expression «observer un électron dont la trajectoire n'est pas modifiée par l'observation » est donc une expression dénuée de toute signification, parce que contradictoire. Voilà pour le mystère!

Reste que la relation d'indétermination de Heisenberg a des incidences épistémologiques très profondes. Elle a d'abord permis une compréhension plus exacte de la nature de l'instrument. Les instruments de la physique classique sont très grossiers, même les plus délicats d'entre eux. Du moins, ils ne sont opératoires que face à des masses et des énergies relativement importantes, et leur seuil de sensibilité reste élevé. Une balance permettant de peser des masses de l'ordre du kilogramme au milligramme près serait une balance très précise. La mesure qu'elle donnerait serait dite «exacte» et en effet elle le serait, puisque cette balance serait fonctionnellement incapable de déceler sa propre erreur. 
D'autre part, la physique classique présuppose toujours une disproportion entre le phénomène observé et l'instrument utilisé pour observer ce phénomène. Je peux me servir d'un thermomètre qui pèse quelques grammes, pour mesurer la température ambiante de cette pièce, parce qu'il y a une disproportion entre la masse d'air dont je mesure la température et la masse du thermomètre. Ainsi, quelle que soit la température initiale et la chaleur spécifique de mon thermomètre, il n'a aucune influence décelable sur la température de l'air. Mais si, par souci de précision, je fabriquais un énorme thermomètre, dont la colonne de mercure permettrait des lectures au millième de degré près, la masse de ce thermomètre, sa température initiale et sa chaleur spécifique auraient une influence notable sur la température de la pièce et, quelle que soit la précision de l'instrument, il n'enregistrerait avec précision qu'une température moyenne: la température de la pièce modifiée par la quantité de chaleur apportée ou soustraite par l'introduction du thermomètre.

Or, dans le monde à notre échelle, qui est le monde de la physique classique, cette disproportion entre le phénomène et l'instrument est aisément respectée : la perturbation apportée par l'instrument reste presque toujours indécelable. De là provient cette conception propre à la physique classique - et au sens commun - de l'observateur impartial, le sujet, qui enregistre, mais n'intervient pas dans le déroulement du phénomène, l'objet. De là, donc, ce dualisme radical entre le Sujet et l'Objet. Ce dualisme n'était pas posé comme postulat, il n'était pas imposé comme dogme, il était une «évidence».

Pourtant dans certains domaines, tel celui de l'optique, une limitation essentielle des possibilités expérimentales avait déjà dû être reconnue. Ainsi dans le cas de la vision, le transfert des informations entre l'objet vu et la rétine s'opère par la lumière. Sans instrument, notre oeil ne perçoit que les images d'objets dont les dimensions sont très grandes par rapport à la longueur d'onde de la lumière. Avec l'invention du microscope on put voir des objets beaucoup plus petits, mais il est évident qu'on ne peut voir un objet dont les dimensions seraient de l'ordre 
de la longueur d'onde de la lumière utilisée. La longueur d'onde de la lumière impose donc une limite essentiellement infranchissable aux possibilités de la vision. Ce fait est d'autant plus intéressant qu'il est techniquement possible de réaliser des microscopes qui grossiraient plusieurs milliers de fois, mais de tels microscopes seraient inutilisables à cause de la nature ondulatoire de la lumière. Aussi, la proposition «voir un objet dont les dimensions sont de l'ordre de quelques angströms» est une proposition contradictoire, et n'a aucun sens.

Il est normal que cette limitation des possibilités d'expérience n'apparut que très tardivement dans le développement de la physique. Ce n'est en effet qu'au niveau de l'atome que toute intervention instrumentale s'accompagne de transformations énergétiques qui sont nécessairement du niveau énergétique des phénomènes que l'on voudrait observer, et ce à cause de l'ordre de grandeur des phénomènes sous observation.

Nous voyons donc que, sur le plan instrumental, il n'y a pas de cassure, ou de révolution de la physique quantique par rapport à la physique classique. La physique classique, comme la physique quantique, était essentiellement limitée dans ses possibilités expérimentales, mais elle n'avait pas eu l'occasion d'en prendre conscience. Par conséquent, le physicien n'a jamais été «face» à la nature; au contraire, il a toujours été en dialogue avec elle, suivant l'expression de Heisenberg. Le dualisme sujetobjet n'a jamais été qu'une vue de l'esprit, ou une absolutisation due à notre façon de penser, et provoquée par le fait que, pendant des millénaires, le «dialogue» s'effectuait entre des pôles tellement éloignés qu'on ne pouvait concevoir qu'il s'agissait bien d'un dialogue plutôt que d'une dichotomie.

$$
\text { * * * }
$$

Prenons maintenant comme point de départ, un domaine apparemment très éloigné de celui que nous venons de quitter, celui de la psychologie.

On connaît la thèse de Watson qui identifie le «moi » et le comportement. Cette thèse behavioriste fut critiquée par plusieurs, entre autres par Warren, qui voyait dans cette identifica- 
tion, une confusion. Watson se serait rendu coupable, aux dires de Warren, de ce que ce dernier appelait le «sophisme du thermomètre »: Warren maintenait que le comportement n'était qu'un révélateur ou un indicateur, que par conséquent ne pas distinguer le comportement, du moi qu'il révèle, c'est comme si un physicien confondait le thermomètre avec la température que celui-ci mesure ${ }^{6}$.

Nous voici donc revenu à une question d'instrumentation : une analyse plus poussée de ce «sophisme » nous permettra sans doute de mieux cerner ce qu'est un instrument.

A première vue, il est évident que le physicien distingue l'instrument et le phénomène observé. On dira que la température est «autre chose» que la dilatation de la colonne de mercure, et on apportera comme preuve que si un premier thermomètre se brise, on pourra en utiliser un autre, ce qui indique que la réalité de la température n'était pas dépendante de l'existence du premier thermomètre. Mais si par sortilège tous les thermomètres, de tout modèle et de tout type se brisaient? Excellente affaire pour tous les fabricants de thermomètre, dirat-on, ce qui est une preuve de plus qu'entre temps la température n'a jamais cessé d'exister. D'ailleurs, dans l'intervalle, les choses ont toujours continué d'être chaudes ou froides quand je les touche, les poulets n'ont pas cessé de cuire, l'eau de s'évaporer, les rails de se dilater et les trains de dérailler. S'il m'avait été absolument nécessaire de mesurer la température, j'aurais pu le faire en chronométrant le temps de cuisson du poulet, ou même en consultant les statistiques des déraillements ferroviaires.

Mais allons plus loin, et supposons que par un imprévisible coup de sort aucun de ces phénomènes habituellement liés à la température ne se produise plus. La température existera-t-elle toujours? Et dans ce cas, où donc se sera-t-elle réfugiée? N'est-il pas clair, au contraire que la température n'est pas autre chose

6. Nous généralisons à dessein la critique faite par Warren, car celui-ci, behavioriste comme Watson, affirme seulement que le comportement est révélateur d'un ensemble de réactions neuro-physiologiques, et non d'un «moi». Cfr. à ce sujet : André Tilquin, Le Bebaviorisme. Paris, Vrin, 1950, p. 421 et ss. 
que la somme de ses phénomènes? Il se fait cependant que certains parmi ces phénomènes sont plus marquants que d'autres, ou plus facilement observables et partant plus facilement mesurables. Il y a de fortes chances dans ce cas que l'un ou l'autre de ces «phénomènes plus marquants» retienne l'attention d'un physicien et, qu'ayant été étalonné, il reçoive le nom d' «instrument». Il apparaît donc que l'instrument même classique, par sa nature, n'est jamais «en dehors» du phénomène qu'il me permet d'observer, il se confond avec ce phénomène, tout au plus peut-on dire - mais c'est là un point de vue subjectif qu'il est un aspect exemplaire du phénomène.

Ainsi, nous voyons s'évanouir le dualisme de l'instrument et du phénomène, car le seul fondement de ce dualisme repose sur la subjectivité qui ayant fixé son attention sur un phénomène, l'isole mentalement et l'absolutise. C'est la condition nécessaire et suffisante pour que nous puissions considérer l'instrument d'observation d'une part, et le phénomène observé d'autre part. Mais cette condition est subjective.

Sans doute l'exemple de la température sera-t-il assez facilement accepté, mais il n'en sera probablement pas de même de la généralisation faite à partir de ce seul exemple. Ainsi dira-t-on, la cellule p'otoślectrique enregistre l'intensité de la lumière, mais ni elle-même, ni son mécanisme ne peuvent être assimilés à des «phénomènes lumineux ». Le thermomètre n'enregistre peut-être que sa propre température, mais il est absurde de dire que la cellule photoélectrique «enregistre sa propre lumière». A y regarder de plus près, cependant, cette absurdité s'affaiblit considérablement; en effet, elle est une absurdité conceptuelle seulement. Elle naît de ce que l'intelligence est forcée de penser par concept. L'intelligence ne pensera clairement que si elle utilise des concepts clairs, et des concetps clairs sont des concepts tranchés, délimités.

C'est ainsi que nous conceptualisons un «phénomène» comme s'il était un «événement isolé ». Or, qu'est-ce que cela peut bien vouloir dire, un «événement isolé»? N'est-il pas clair, au contraire, que ce n'est que mentalement qu'un phénomène est isolable? Par conséquent, entre la position de l'aiguille 
d'un photomètre et l'activité de la source lumineuse, il y a une succession ininterrompue d'événements, mais, pour y voir clair, notre intelligence se sent forcée de sectionner cette succession en phénomènes distincts, que nous nommerons «activité des électrons», «rayonnement lumineux », «impact des photons», etc. Nous devons donc élargir encore notre conclusion précédente: non seulement la distinction entre le «phénomène observé » et le «phénomène-instrument » est-elle une distinction conceptuelle, et non réelle, mais il en va de même pour les distinctions de phénomènes à phénomènes...

$$
\text { * * * }
$$

Il est temps, après ce long détour, de revenir à Heisenberg. Celui-ci maintient que l'homme n'est pas face à la nature, mais en dialogue avec elle, voulant dire par là que l'homme ne peut d'aucune manière connaître la nature en soi. Toute tentative en ce sens est vouée à l'échec, car elle impliquerait une intervention de l'homme dans le cours régulier des choses.

Cette conception est plus justifiée que celle du dualisme cartésien, mais elle postule que l'intervention de l'homme soit un phénomène d'un «autre ordre » que l'ordre du cours régulier de la nature, ce qui nous rapproche du dualisme cartésien. D'autre part, sans ce postulat, l'intervention de l'homme est un phénomène nullement irrégulier, et dans ce cas, la déviation de l'électron observé redevient un phénomène tout aussi objectif que n'importe quel phénomène où l'homme n'intervient pas. Sans ce postulat, encore, c'est l'homme lui-même qui devient un ensemble de phénomènes, comme le dirait, par exemple et parmi bien d'autres, Jacques Monod: «L'analyse objective nous oblige à voir une illusion dans le dualisme apparent de l'être ${ }^{7}$. Dans ce cas, toujours, c'est la distinction même entre Sujet et Objet, qui s'évanouit, ces deux pôles étant intégrés dans une objectivité plus haute, ce que nous appelons le monisme objectiviste.

Mais, objectera-t-on encore, la subjectivité n'est nullement postulée par Heisenberg, au sens arbitraire et gratuit de ce terme. Au contraire la subjectivité s'impose inévitablement, c'est la né-

7. J. MONOD, Le Hasard et la Nécessité. Éd. du Seuil, Paris, 1970, p. 173. 
gation de la subjectivité qui est impossible. On ne manquera pas de relever que cet exposé même fait sans cesse appel à la subjectivité : c'est le physicien qui, ayant fixé son attention sur un phénomène remarquable, le choisit pour l'utiliser comme phénomène-instrument, c'est la pensée humaine qui distingue les phénomènes observés et l'instrument qui permet d'observer, ce n'est que par la pensée que nous pouvons isoler certains événements dans une séquence continue etc... Eddington exprime cette inévitabilité de la subjectivité beaucoup mieux que moi, et de façon beaucoup plus autorisée : "Là où la science est allée le plus loin, l'esprit a simplement regagné sur la nature ce qu'il y avait investi. Aux frontières de l'inconnu, nous avons découvert des empreintes étranges. Nous avons inventé des théories exhaustives pour élucider leur origine. Enfin, nous sommes parvenus à reconstituer la créature qui les a laissées. Et voilà que ce sont nos propres empreintes ${ }^{8}$. C'est là une expression imagée, et belle, du monisme subjectiviste.

$$
* * *
$$

Une critique du dualisme cartésien nous avait conduit, par la notion de dialogue, chère à Heisenberg, au monisme objectif de Monod. Mais voilà que, sans un moment de répit, nous sommes forcés d'embrasser le subjectivisme le plus radical.

«Tout est objet», «Tout est sujet»: il nous faudrait pourtant choisir.

Mais le choix a-t-il un sens ? Confrontés par deux monismes exclusifs, nous nous trouvons dans l'illusion du devoir choisir, mais ce n'est qu'une illusion, car ce n'est que dans leur formulation que ces monismes s'opposent. En réalité, ils sont équivalents, ils sont identiques : il n'y a, il ne peut y avoir qu'un seul monisme. En effet, toute qualification que l'on voudrait donner au monisme ne peut se faire que par des concepts qui supposent la réalité d'au moins deux entités distinctes et opposées. Or, et par définition, le monisme exclut de telles distinctions. En d'autres mots, ce n'est que dans une conception dualiste que les couples Esprit-

8. A. EDDINGTON, cité par J. de Ries. Réflexions sur les principes de la physique, in Bulletin de Société Royale belge des Ingénieurs et des Industriels, no $9 / 10,1963$, pp. $402-413$. 
Matière, Sujet-Objet peuvent avoir une signification. Par conséquent, des propositions comme «Tout est sujet» ou «Tout est matière », ne peuvent avoir de sens, car elles tentent d'exprimer un monisme en un langage dualiste; elles postulent ce qu'elles rejettent. Il apparaît par là que le monisme n'est pas impossible, mais qu'il est, au sens strict, impensable. Il apparaît que notre problème n'a jamais été qu'un faux problème puisqu'il ne surgissait que de la mauvaise utilisation de certains concepts.

Mais on objectera qu'une utilisation de concept, même mauvaise, suppose un sujet qui pense ces concepts, et donc une autonomie du sujet. D'ailleurs, le monisme, dont je viens de dire qu'il est impensable, a, de fait, été pensé. Et il a été pensé dans un langage dualiste. Quelles qu'aient été les erreurs du langage et de la pensée dualistes, elles n'ont été possibles que moyennant la distinction du sujet et de l'objet, moyennant, donc, la négation du monisme. Nous voici donc ramenés au «si enim fallor, sum» et au cogito cartésien. Mais alors? Descartes? Heisenberg? Monod ? Eddington? ... la ronde infernale reprend de plus belle, et n'aura jamais de fin...

Université de Sherbrooke 